\title{
Results of an intercomparison for electric field strength measurements within the German calibration service
}

\author{
Reiner Pape ${ }^{1}$, Uwe Karsten ${ }^{2}$, Frank-Michael Lindner ${ }^{3}$, Frank Rittmann ${ }^{4}$, Joachim von Freeden ${ }^{5}$, \\ Thomas Kleine-Ostmann ${ }^{1}$, and Thorsten Schrader ${ }^{1}$ \\ ${ }^{1}$ Physikalisch-Technische Bundesanstalt (PTB), Bundesallee 100, 38116 Braunschweig, Germany \\ ${ }^{2}$ TESEQ GmbH, Landsberger Str. 255, 12623 Berlin, Germany \\ ${ }^{3}$ Kalibrierzentrum der Bundeswehr, Sielower Landstr. 66, 03044 Cottbus, Germany \\ ${ }^{4}$ steep GmbH, Lise-Meitner-Strasse 6, 85521 Ottobrunn, Germany \\ ${ }^{5}$ Narda Safety Test Solutions GmbH, Sandwiesenstr. 7, 72793 Pfullingen, Germany
}

Correspondence to: Thomas Kleine-Ostmann (thomas.kleine-ostmann@ptb.de)

Received: 21 December 2016 - Revised: 2 August 2017 - Accepted: 22 September 2017 - Published: 25 October 2017

\begin{abstract}
In this paper we discuss the results of an intercomparison for electric field strength measurements within the German Calibration Service (Deutscher Kalibrierdienst - DKD). The comparison has been carried out on the field strength value required to reach a display reading of $20 \mathrm{~V} \mathrm{~m}^{-1}$ of the field probes for frequencies between $100 \mathrm{MHz}$ and $18 \mathrm{GHz}$. Five laboratories joined the intercomparison including the Physikalisch-Technische Bundesanstalt (PTB), the German National Metrology Institute that keeps the primary standard for electric field strength. As measurement artefacts both a small 1-axis probe usually used as transfer sensor at PTB and a larger 3-axis commercial field probe have been used. While the results agree well for the small field probe and when the larger commercial 3-axis field probe is oriented in the direction of the magnetic field, larger deviations occur, when the larger 3-axis field probe is oriented into the direction of the Poynting vector of the calibration field.
\end{abstract}

\section{Introduction}

Field probes are widely used to determine the electric field strength, e.g. for guaranteeing personal safety in electromagnetic fields or for setting the test levels during electromagnetic compatibility interference testing. Both fields of application require accurate knowledge of the calibration factor of the field probe to be able to measure field strengths traceable to the SI units with known measurement uncertainty. In
Germany, three laboratories are accredited for electric field strength measurements by the DAkkS - Deutsche Akkreditierungsstelle, the national accreditation body for the Federal Republic of Germany. To assess the technical competence of the accredited laboratories, an intercomparison was organised by the Physikalisch-Technische Bundesanstalt (PTB), the German National Metrology Institute, within the framework of the German Calibration Service (Deutscher Kalibrierdienst - DKD), which is the association of the accredited laboratories in Germany (PTB-Mitteilungen, 2015).

In addition to PTB, three accredited and one nonaccredited laboratories took part in the intercomparison. As measurement artefacts both a small 1-axis probe developed by PTB and a commercially available field probe ETSLindgren HI- $6053^{\mathrm{TM}}$ were used in different orientations. The field strength values to generate a reading of $20 \mathrm{~V} \mathrm{~m}^{-1}$ were compared. A field strength reading of $20 \mathrm{~V} \mathrm{~m}^{-1}$ represents a commonly measured field strength value in personal safety assessment and electromagnetic compatibility testing and is well above the display fluctuations observed at zero field strength. The required field strength values to generate readings of $20 \mathrm{~V} \mathrm{~m}^{-1}$ differ for the different field probes and orientations. The 1-axis probe oriented parallel to the magnetic field (PH orientation) was used for a comparison between 300 and $1000 \mathrm{MHz}$ in $100 \mathrm{MHz}$ steps and for $1000 \mathrm{MHz}$ to $6 \mathrm{GHz}$ in $500 \mathrm{MHz}$ steps. The field probe $\mathrm{HI}-6053^{\mathrm{TM}}$ was oriented both parallel to the magnetic field (PH orientation) and parallel to the power flux density vector (PS orientation). It was used for a compari- 


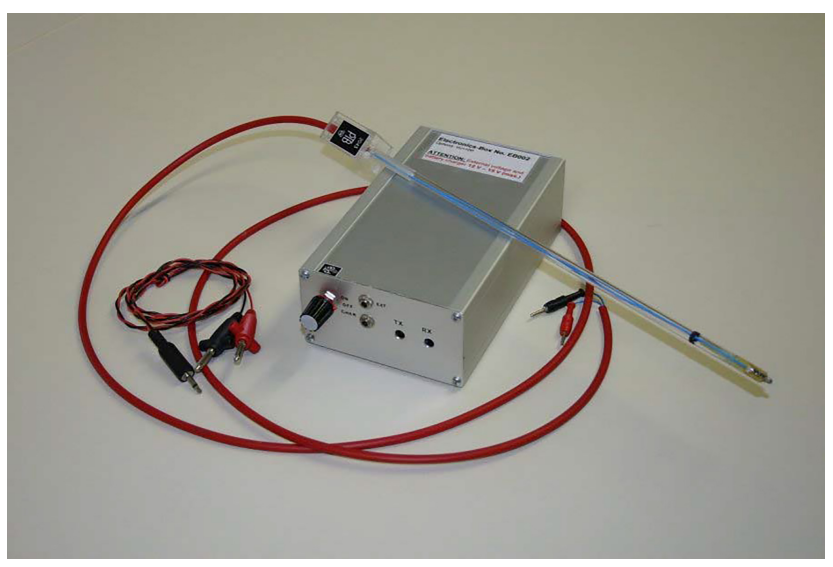

Figure 1. Small 1-axis probe "Transfer sensor" with electronic box for dissemination of field strength developed by the PhysikalischTechnische Bundesanstalt.

son between 100 and $1000 \mathrm{MHz}$ in $100 \mathrm{MHz}$ steps and for $1000 \mathrm{MHz}$ to $18 \mathrm{GHz}$ in $500 \mathrm{MHz}$ steps. The intercomparison shows that all laboratories are able to generate electromagnetic fields with the specified measurement uncertainty but that calibrations in PS orientation can be problematic. The results are supplemental to the outcome of the international key comparison CCEM.RF-K24.F (Eiø et al., 2013) that indicates a problem with field representation for some National Metrology Institutes, whereas this national intercomparison suggests problems with dissemination in some cases. The outcome of this comparison was one of the triggers for re-establishing the standardization working group DKE GAK 767.4.3 "Feldsondenkalibrierung" which works on a first draft on a new standard IEC 61000-4-26 "Field Probe Calibration". The goal of this working group is to overcome the shortcomings identified in current standards and technical guidelines such as IEEE Std $1309^{\mathrm{TM}}-2013$ (IEEE 1309, 2013), IEC 61000-4-3:2006 (IEC 61000-4-3, 2006) and VDI/VDE/DGQ/DKD 2622 Blatt 10 (VDI/VDE/DGQ/DKD 2622, 2004) by targeting all aspects of field probe calibration required for accurate measurements, including field generators, calibration process and the application of the field probe.

In this paper we describe the travelling standards, the intercomparison schedule and the approach taken for data evaluation in Sect. 2. In the following Sects. 3 and 4 we then present the results for the two different types of field probes. In the final section we draw conclusions regarding the calibration procedures for field probes.

\section{Intercomparison}

\subsection{Travelling standards}

As measurement artefacts both a small 1-axis probe (see Fig. 1) and a commercially available 3 -axis field probe ETS-

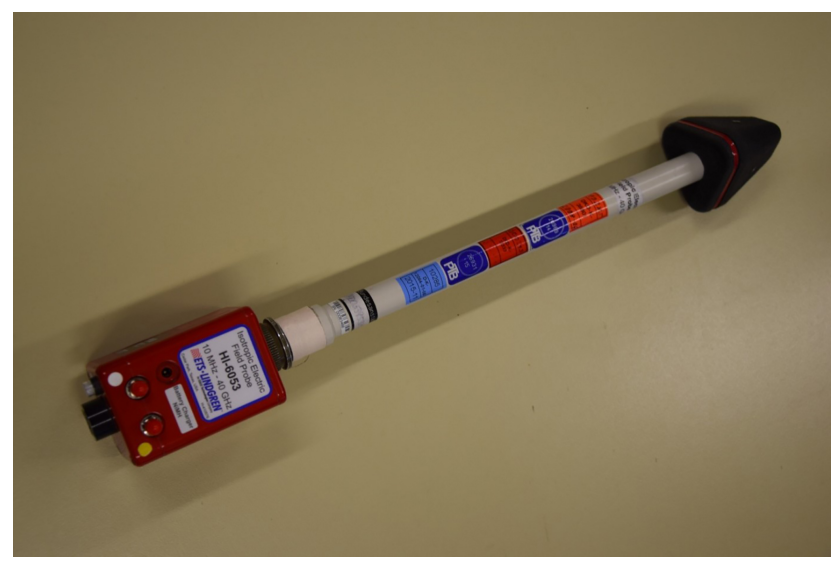

Figure 2. ETS-Lindgren HI- $6053^{\mathrm{TM}} 3$-axis field probe.
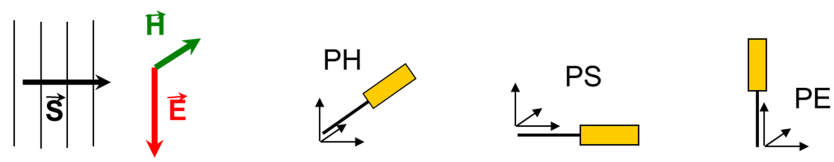

Figure 3. Main orientations of the field probe axis parallel to the magnetic field vector (PH), the Poynting vector (PS) and the electric field vector (PE) of the electromagnetic wave. The yellow box with handle represents the field probe.

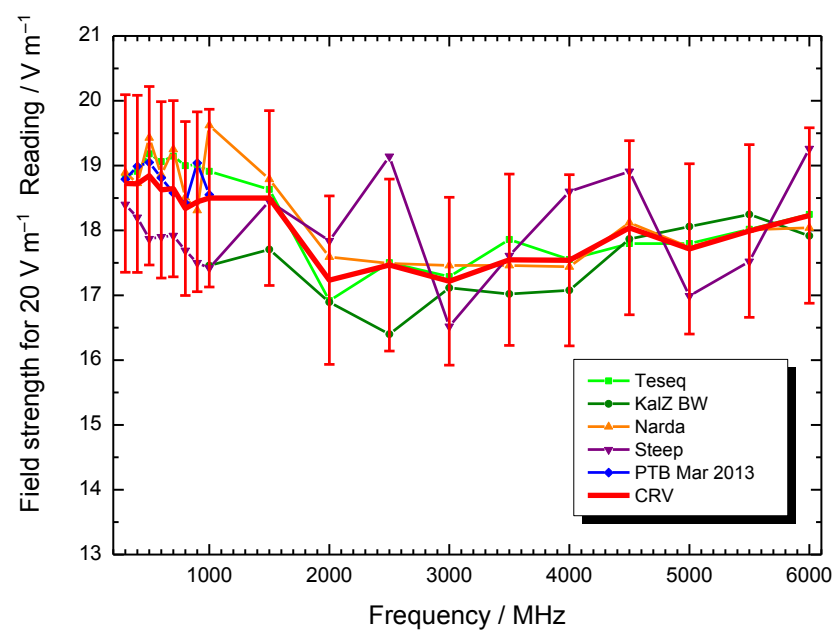

Figure 4. Results of intercomparison (field strength values provided by the participating laboratories and CRV with uncertainty of CRV plotted as error bars) for the transfer sensor oriented in PH direction.

Lindgren HI-6053 ${ }^{\mathrm{TM}}$ (see Fig. 2) were used. The small 1axis probe was developed by PTB and is commonly used as a transfer sensor to establish traceability for larger field generators. Here, it is chosen as it minimizes the field perturbation during measurement and provides reasonable information about the field generation without larger influence from the detector. The commercial field probe ETS-Lindgren HI$6053^{\mathrm{TM}}$ represents a typical 3 -axis field probe with the sen- 
(a)

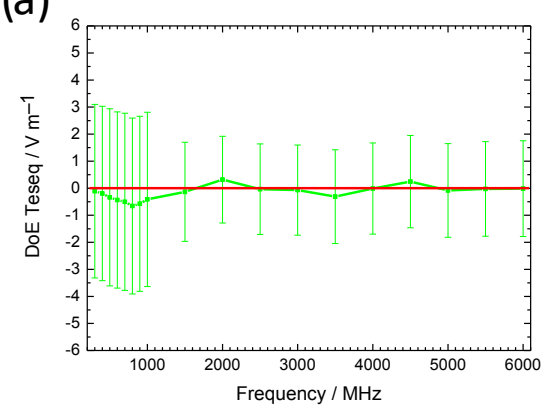

(b)

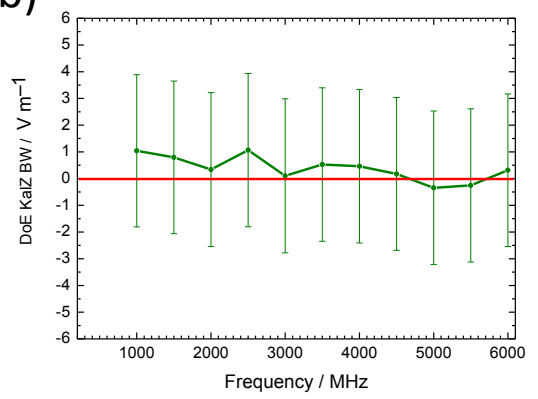

(c)

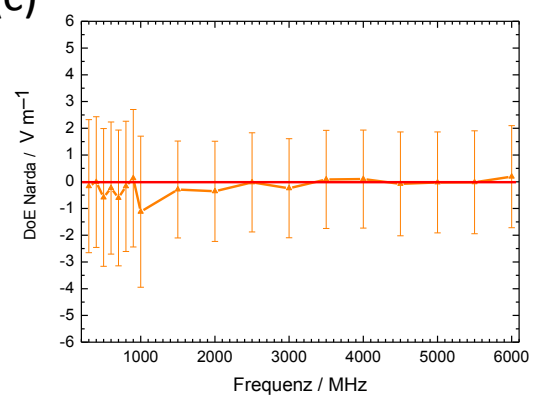

(d)

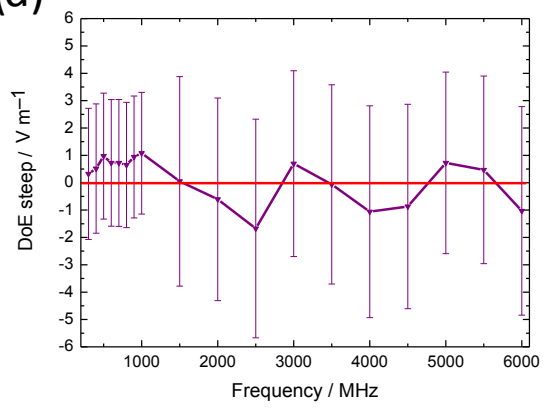

(e)

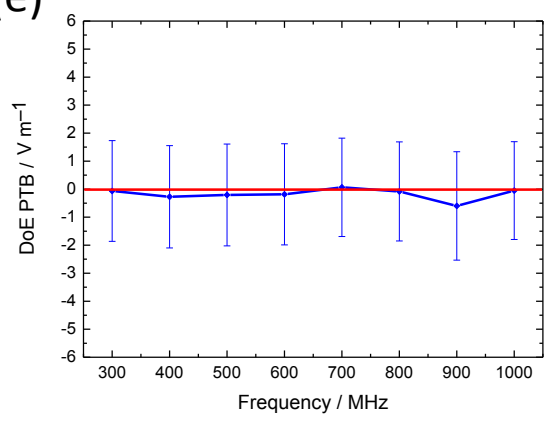

Figure 5. Results of intercomparison in terms of DoE and $U(\mathrm{DoE})$ for the different participants and measurement constellations for the transfer sensor in PH orientation. (a) Teseq, (b) KalZ BW, (c) Narda, (d) steep, and (e) PTB.

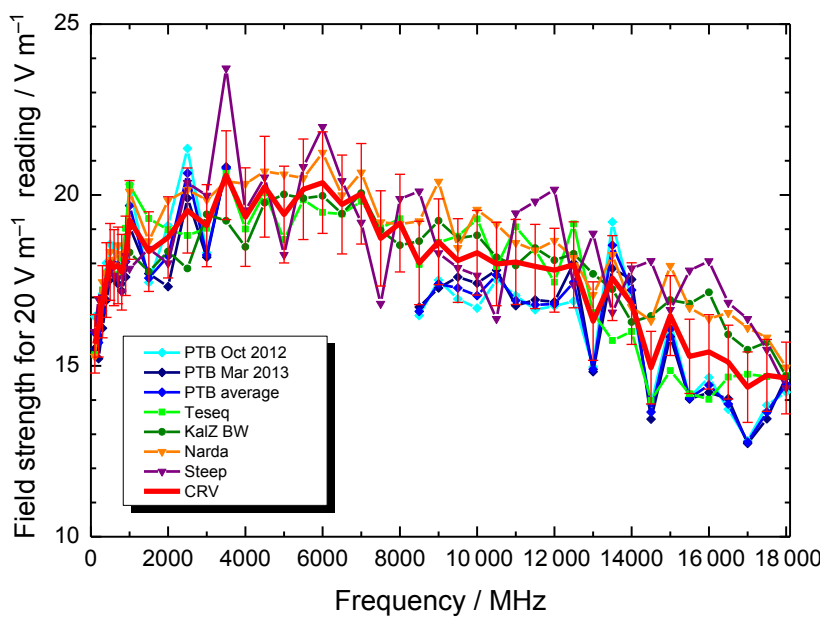

Figure 6. Results of intercomparison (field strength values provided by the participating laboratories and CRV with uncertainty of CRV plotted as error bars) for the ETS-Lindgren HI-6053 ${ }^{\mathrm{TM}} 3$-axis field probe in $\mathrm{PH}$ orientation.

sor head connected to the readout unit by a rod. Such probes need to be calibrated in accordance with their application in electromagnetic compatibility testing or personal safety measurements. The main orientations of the field probe with regard to the electromagnetic field and its propagation direction are denoted with PE, PS and PH as shown in Fig. 3.
The field strength values to generate a reading of $20 \mathrm{~V} \mathrm{~m}^{-1}$ at the instruments were compared. The transfer sensor in $\mathrm{PH}$ orientation was used for a comparison between 300 and $1000 \mathrm{MHz}$ in $100 \mathrm{MHz}$ steps and for $1000 \mathrm{MHz}$ to $6 \mathrm{GHz}$ in $500 \mathrm{MHz}$ steps. The field probe $\mathrm{HI}-6053^{\mathrm{TM}}$ was oriented both in PH and PS directions. It was used for a comparison between 100 and $1000 \mathrm{MHz}$ in $100 \mathrm{MHz}$ steps and for $1000 \mathrm{MHz}$ to $18 \mathrm{GHz}$ in $500 \mathrm{MHz}$ steps.

\subsection{Time schedule}

The field probes were first measured at PhysikalischTechnische Bundesanstalt (PTB) in Braunschweig, then at TESEQ GmbH (Teseq) in Berlin, then at the Kalibrierzentrum der Bundeswehr in Cottbus (KalZ BW), after that at Narda Safety Test Solutions GmbH, in Pfullingen (Narda), then at steep GmbH in Ottobrunn (steep) and finally at PTB again.

The measurement campaign took place from October 2012 until March 2013.

\subsection{Data evaluation}

The intercomparison was evaluated based on the measurement results reported by all laboratories that were able to calibrate the distinct field probes. According to Cox (2002) a comparison reference value $C R V$ and its uncertainty $U(\mathrm{CRV})$ were calculated based on the field strength values $E_{i}$ and the 
(a)

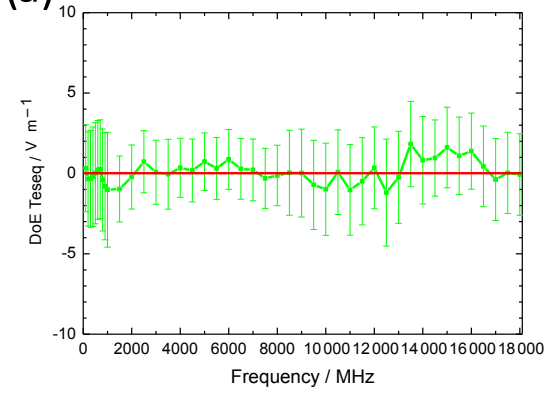

(b)

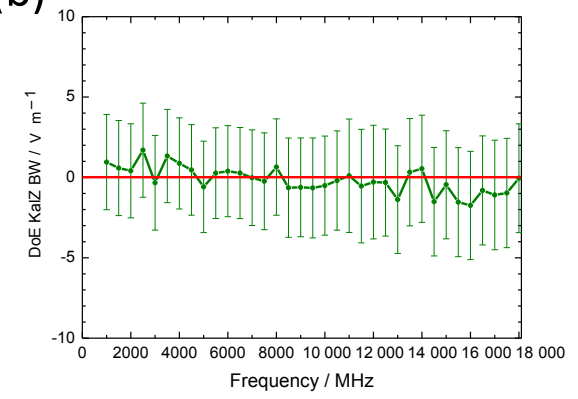

(c)

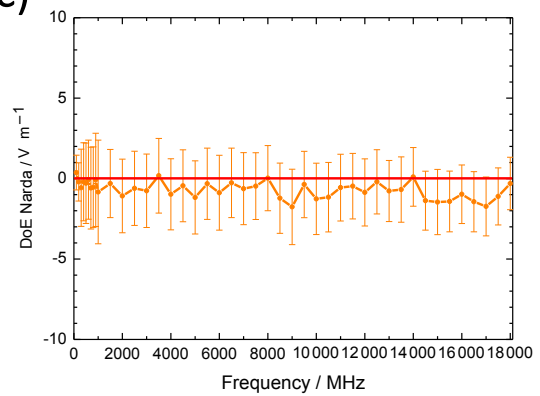

(d)

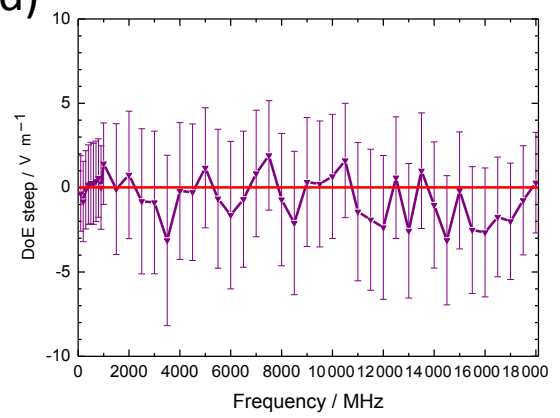

(e)

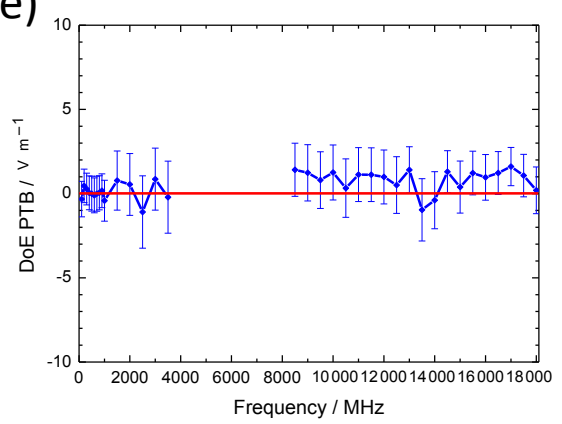

Figure 7. Results of intercomparison in terms of DoE and $U(\mathrm{DoE})$ for the different participants and measurement constellations for the ETS-Lindgren HI-6053 ${ }^{\mathrm{TM}} 3$-axis field probe in PH orientation. (a) Teseq, (b) KalZ BW, (c) Narda, (d) steep, and (e) PTB.

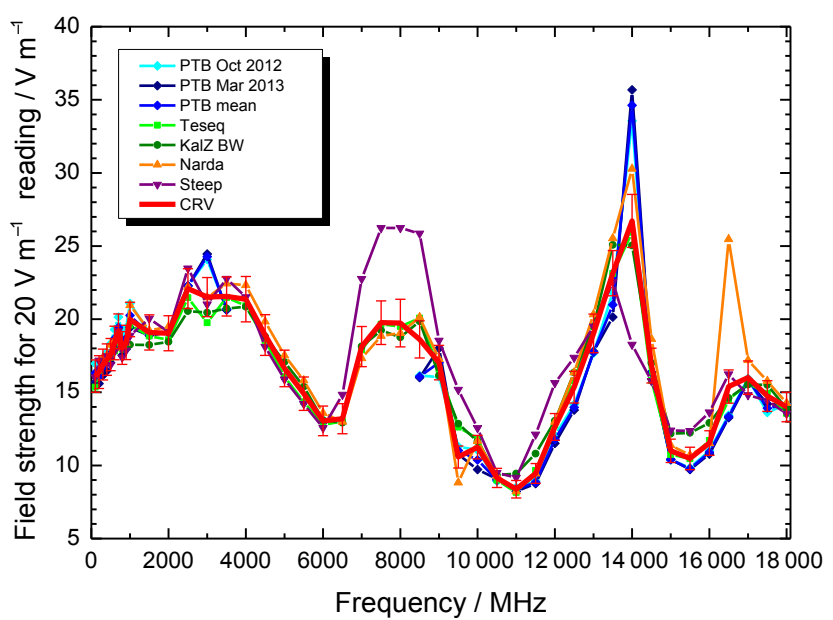

Figure 8. Results of intercomparison (field strength values provided by the participating laboratories and CRV with uncertainty of CRV plotted as error bars) for the ETS-Lindgren HI- $6053^{\mathrm{TM}} 3$-axis field probe in PS orientation.

measurement uncertainties $U\left(E_{i}\right)$ reported by the individual laboratories in accordance with the "Guide to the expression of uncertainty in measurement” (GUM, 1995):

$\mathrm{CRV}=\sum_{i} w_{i} \cdot E_{i}$ with $w_{i}=\left(\sum_{i} \frac{1}{U^{2}\left(E_{i}\right)}\right)^{-1} \cdot \frac{1}{U^{2}\left(E_{i}\right)}$
$U(\mathrm{CRV})=\sqrt{\sum_{i} w_{i}^{2} U^{2}\left(E_{i}\right)}$

The weighting factor $w_{i}$ results in a higher contribution of the laboratories with lower uncertainties to comparison reference value CRV.

From the field strength values $E_{i}$ and the measurement uncertainties $U\left(E_{i}\right)$ reported by the individual laboratories and the comparison reference value CRV a degree of equivalence DoE and its uncertainty $U(\mathrm{DoE})$ has been calculated using

$\mathrm{DoE}_{i}=\mathrm{CRV}-E_{i}$

$U\left(\mathrm{DoE}_{i}\right)=\sqrt{U^{2}\left(E_{i}\right)-U^{2}(\mathrm{CRV})}$

as a measure for the deviation from the comparison reference value for each laboratory. A deviation of a single calibration result from the consensus value CRV that cannot be explained by the reported measurement uncertainties can be easily observed when plotting the DoE and its uncertainty. In that case the absolute value of the DoE is larger than its uncertainty. The calculation is based on expanded uncertainties so that a confidence interval of $95 \%$ applies.

The measurement results from PTB were treated in the same way as those of the other participants, neglecting the fact that PTB provides traceability to some of the other laboratories in some of the frequency ranges used in this intercomparison. However, we assume that the errors due to 
(a)

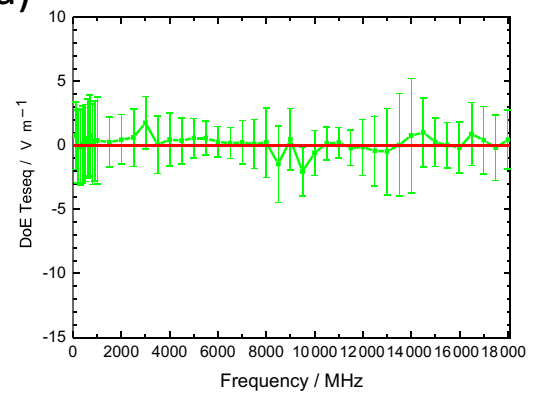

(b)

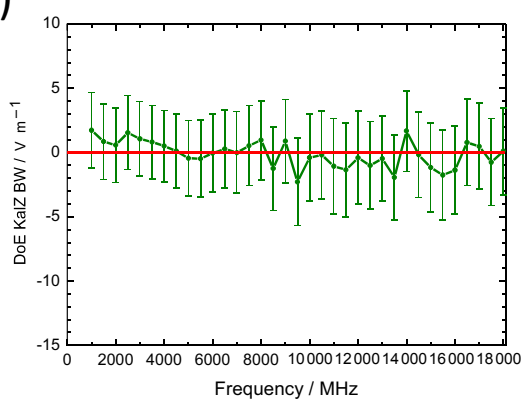

(c)

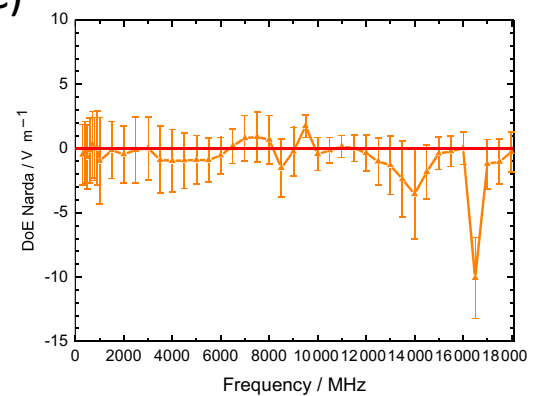

(d)

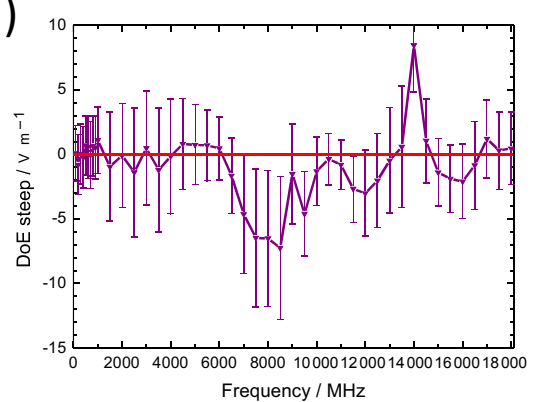

(e)

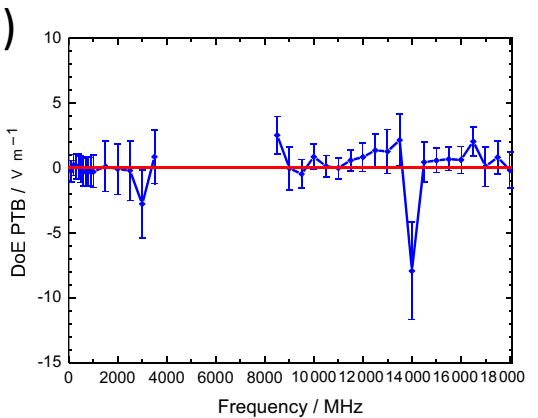

Figure 9. Results of intercomparison in terms of DoE and $U$ (DoE) for the different participants and measurement constellations for the ETS-Lindgren HI-6053 ${ }^{\mathrm{TM}}$ 3-axis field probe in PS orientation. (a) Teseq, (b) KalZ BW, (c) Narda, (d) steep, and (e) PTB.

correlation are rather low. In case both measurements at the beginning and at the end were available from PTB, the mean value was used as input to the data evaluation. As no drifts of the measurement artefacts could be identified, a drift correction was not applied.

\section{Results for the small 1-axis probe}

The calibration results for the transfer sensor are shown in Fig. 4. From PTB calibration data was available for a limited frequency range from 300 to $1000 \mathrm{MHz}$ at the end of the campaign, only, due to technical limitations. The degrees of equivalence for the individual laboratories are shown in Fig. 5. The results of all laboratories agree well with the calculated CRV within $U(\mathrm{DoE})$ calculated from the specified measurement uncertainty of the laboratory.

\section{Results for the 3-axis commercial field probe}

In Fig. 6 the calibration results for the field probe ETSLindgren $\mathrm{HI}-6053^{\mathrm{TM}}$ in $\mathrm{PH}$ orientation are shown. The corresponding degrees of equivalence for the individual laboratories are shown in Fig. 7. Due to technical reasons, PTB calibration data was not available from 4000 to $8000 \mathrm{MHz}$, whereas calibration data was not available from KalZ BW from 100 to $900 \mathrm{MHz}$. The results of all laboratories agree with the calculated comparison reference value within their specified measurement uncertainties at nearly all frequency points.

The calibration results for the field probe ETS-Lindgren HI- $6053^{\mathrm{TM}}$ in PS orientation are shown in Fig. 8. The corresponding degrees of equivalence for the individual laboratories are shown in Fig. 9. As in the case of PH orientation of the probe, PTB calibration data was not available from 4000 to $8000 \mathrm{MHz}$, whereas calibration data was not available from KalZ BW from 100 to $900 \mathrm{MHz}$. It can be seen, that the calibration results of three laboratories do not agree with the calculated comparison reference value considering their specified measurement uncertainties at distinct frequencies around 8000,14000 and $16500 \mathrm{MHz}$ (if this is a problem of the three laboratories, only, is not clear until the input quantities are coherently identified. It is unlikely that the CRV represents the true value, here). As can be seen from Fig. 8, these frequencies correspond to oscillations in the calibration factor, which can be attributed to standing waves in front of the field probe (Kleine-Ostmann et al., 2007). Clearly, the measurement uncertainty is underestimated at the affected frequencies when calibrating a field probe in PS orientation with large housing that causes standing waves. Assuming the result changes noticeable by an angular misalignment within a few degrees the laboratory will not be able to declare the uncertainty with good confidence until the angle dependence has been determined. 


\section{Conclusion}

Generally results agree well for the small field probe and when the larger commercial field probe is oriented in the direction of the magnetic field. In these cases calibration curves are reasonably flat, as no problems with standing waves in front of the field probe occur. The very good agreement between the laboratories shows that the specified measurement uncertainties are realistic and that no general problem with the representation of field strength exists. However, when it comes to dissemination, the calibration of larger field probes can be problematic at distinct frequencies when standing waves occur due to orientation of the field probe in the direction of the Poynting vector. It is very important that calibration laboratories identify these problems in order to maintain their specified calibration uncertainties or to take measures for a wise consideration of such calibration results into the uncertainty budget. Some calibration laboratories changed their calibration setups already, so that the situation described in this intercomparison might have changed by now. The findings will directly contribute to a new standard IEC 61000-4-26 "Field Probe Calibration" for which a first draft is under development in the standardization working group DKE GAK 767.4.3 "Feldsondenkalibrierung".

Data availability. The underlying data sets are property of the participating laboratories and are not made available to the public.

Competing interests. The authors declare that they have no conflict of interest.

Edited by: Michael Vogt

Reviewed by: two anonymous referees

\section{References}

Cox, M. G.: The evaluation of key comparison data, Metrologia, 39, 589-595, 2002.

Eiø, C., Gentle, D., Fernandez, A., Le Sage, Y., Kleine-Ostmann, T., Camell, D., Borsero, M., Vizio, G., Pythoud, F., Mühlemann, B., Drazil, K., Zhao, D., Ji, Y., Kang, N.-W., Dabo, L., Ming, X., Morioka, T., Hirose, M., Kolotygin, S., Neustroev, S., Cetintas, M., and Sen, O.: Final report on key comparison CCEM.RF-K24.F: E-field measurements at frequencies of $1 \mathrm{GHz}, 2.45 \mathrm{GHz}, 10 \mathrm{GHz}$ and $18 \mathrm{GHz}$ and at indicated field levels of $10 \mathrm{~V} / \mathrm{m}, 30 \mathrm{~V} / \mathrm{m}$ and $100 \mathrm{~V} / \mathrm{m}$, Metrologia, 50, 01007, https://doi.org/10.1088/0026-1394/50/1A/01007, 2013.

GUM: Evaluation of measurement data - Guide to the expression of uncertainty in measurement, JCGM 100:2008, GUM 1995 with minor corrections, Working Group 1 of the Joint Committee for Guides in Metrology (JCGM/WG 1), 1995.

IEC 61000-4-3:2006+AMD1:2007+AMD2:2010 CSV, Consolidated version: Electromagnetic compatibility (EMC) - Part 43: Testing and measurement techniques - Radiated, radiofrequency, electromagnetic field immunity test, International Electrotechnical Commission, Geneva, April 2010.

IEEE Std 1309 ${ }^{\text {TM}}-2013$ : IEEE Standard for Calibration of Electromagnetic Field Sensors and Probes, Excluding Antennas, from $9 \mathrm{kHz}$ to $40 \mathrm{GHz}$, Institute of Electrical and Electronics Engineers, New York, https://doi.org/10.1109/IEEESTD.2013.6673999, November 2013.

Kleine-Ostmann, T., Pape, R., Münter, K., and Schrader, T.: Anisotropie von Feldstärkemessgeräten, Technisches Messen, 74, 521-528, 2007.

PTB-Mitteilungen: The new German Calibration Service (DKD), in: Vol. 124, English Edition, Physikalisch-Technische Bundesanstalt, Braunschweig, April 2015.

VDI/VDE/DGQ/DKD 2622 Blatt 10: Kalibrieren von Messmitteln für elektrische Größen - Hochfrequenz-Feldstärke-Messgeräte, Verein Deutscher Ingenieure, Düsseldorf, June 2004. 